\title{
REGULAR VERSUS DIFFUSIVE PHOTOSPHERIC FLUX CANCELLATION
}

\author{
YURI E. LITVINENKO \\ Department of Mathematics, University of Waikato, P. B. 3105, Hamilton, New Zealand \\ Received 2010 December 28; accepted 2011 March 16; published 2011 March 31
}

\begin{abstract}
Observations of photospheric flux cancellation on the Sun imply that cancellation can be a diffusive rather than regular process. A criterion is derived, which quantifies the parameter range in which diffusive photospheric cancellation should occur. Numerical estimates show that regular cancellation models should be expected to give a quantitatively accurate description of photospheric cancellation. The estimates rely on a recently suggested scaling for a turbulent magnetic diffusivity, which is consistent with the diffusivity measurements on spatial scales varying by almost two orders of magnitude. Application of the turbulent diffusivity to large-scale dispersal of the photospheric magnetic flux is discussed.
\end{abstract}

Key words: magnetic fields - magnetic reconnection - Sun: photosphere

\section{INTRODUCTION}

Photospheric flux cancellation on the Sun had been originally defined as the mutual disappearance of magnetic flux in closely spaced features of opposite polarity (Martin et al. 1985; Livi et al. 1985). Detailed observations and theoretical arguments strongly suggest that cancellation is a manifestation of magnetic reconnection in the solar photosphere (Martin 1990; Litvinenko \& Martin 1999; Chae et al. 2002).

Photospheric magnetic reconnection is interesting both in its own right and as a key process in the formation and evolution of solar filaments (e.g., Martin 1998; Martens \& Zwaan 2001; Wood \& Martens 2003; Martin et al. 2008; Litvinenko 2010; Okamoto et al. 2010), which motivated theoretical work on photospheric magnetic reconnection and its observational consequences (Sturrock 1999; Litvinenko 1999). The traditional Sweet-Parker reconnection model (Parker 1957) has been adapted to the physical parameters of the solar photosphere, and an important effect of magnetic flux pile-up in the reconnection inflow region has been incorporated into the model. The resulting reconnection scalings have been used to interpret observations of photospheric cancellation (Litvinenko et al. 2007; Park et al. 2009, and references therein).

Recent observations, however, do not seem to support this straightforward approach to modeling of photospheric cancellation. High-resolution $\mathrm{SOHO}$ Michelson Doppler Imager (MDI) and Hinode Solar Optical Telescope (SOT) data provide clear evidence that the photospheric velocity and magnetic fields in canceling features have significant fluctuating components (e.g., Chae et al. 2008, and references therein). In particular, the velocity field of interacting magnetic fragments in photospheric canceling features can strongly vary in the course of cancellation (see, e.g., Figure 3 in Litvinenko et al. 2007). The observed variability implies the dominance of small-scale transient flows, as opposed to the more regular and longer-lived supergranular flows that often appear to drive flux cancellation. Analysis of SOHO MDI magnetograms also indicates that opposite-polarity fluxes do not systematically converge everywhere onto the polarity inversion line where cancellation takes place. Instead, the fluxes diffuse toward the polarity inversion line (Wang \& Muglach 2007).

Two regimes of photospheric cancellation can be defined, depending on the amplitudes of random fluctuations in the velocity field and the magnetic field. Regular cancellation is driven by a clearly identifiable convergence of magnetic fragments, in which case the cancellation time is inversely proportional to the convergence speed. By contrast, diffusive cancellation is driven by random motions of the photospheric plasma, in which case neighboring magnetic fragments of opposite polarity could be observed to disappear in the absence of significant regular displacements of the fragments toward each other.

Observational manifestations of photospheric magnetic reconnection generally should depend on both regular and diffusive processes, and quantitative modeling of photospheric flux cancellation may require both processes to be taken into consideration. Yet the photospheric reconnection models developed so far (Litvinenko et al. 2007; Park et al. 2009) assume the presence of a regular plasma flow (for instance, a stagnation-point flow), which transports magnetic fragments of opposite polarity toward each other and drives magnetic reconnection. Possible diffusive effects in cancellation are neglected in this approach.

Motivated by these considerations, the purpose of this note is to derive and discuss a criterion that quantifies the parameter range in which diffusive photospheric cancellation might occur.

\section{A CRITERION FOR DIFFUSIVE CANCELLATION}

Consider two interacting opposite-polarity fragments of the photospheric magnetic flux, and let the $x$-axis coincide with the line connecting the fragment centers. If the initial distance between the fragments is small compared with their sizes, diffusive cancellation can be sufficiently accurately described as one-dimensional diffusion. Suppose the cancellation is driven by a random photospheric flow characterized by the mean square velocity $\left\langle v^{2}\right\rangle$ and the correlation time $\tau$. The displacement variance for either fragment at time $t$ is

$$
\left\langle x^{2}\right\rangle=\left\langle v^{2}\right\rangle \tau t .
$$

When the rate of flux cancellation is controlled by such random velocity flow, the diffusive cancellation time $t_{\mathrm{d}}$ can be defined by the condition $l^{2}=2\left\langle x^{2}\right\rangle$. Here, $l$ is the initial distance between the fragment centers, and the factor of two reflects the fact that both fragments are diffusing. Hence, the diffusive cancellation time is given by

$$
t_{\mathrm{d}} \simeq \frac{l^{2}}{2 \tau\left\langle v^{2}\right\rangle} .
$$


By contrast, given a regular convergence speed $u$ of either fragment, the regular cancellation time is simply

$$
t_{\mathrm{r}} \simeq \frac{l}{2 u} .
$$

The condition $t_{\mathrm{d}}<t_{\mathrm{r}}$ identifies the parameter range in which regular cancellation is dominated by diffusive cancellation:

$$
\frac{u l}{\tau\left\langle v^{2}\right\rangle}<1
$$

A numerical estimate for the critical value of $u$, separating the diffusive and regular cancellation regimes, can now be made once the values of $\tau$ and $\left\langle v^{2}\right\rangle$ are specified. For example, Sturrock \& Uchida (1981), in their analysis of stochastic motion of magnetic field lines at the photospheric level, employed the values of the correlation time $\tau \simeq 2 \times 10^{3}$ s and the photospheric root-mean-square speed $\left\langle v^{2}\right\rangle^{1 / 2} \simeq 10^{5} \mathrm{~cm} \mathrm{~s}^{-1}$. Using these values in the equation above would lead to $u<2 \mathrm{~km} \mathrm{~s}^{-1}$ for $l=10^{8} \mathrm{~cm}$ and $u<0.2 \mathrm{~km} \mathrm{~s}^{-1}$ for $l=10^{9} \mathrm{~cm}$. Here the smaller value of $l$ corresponds to typical sizes of photospheric canceling features on the quiet Sun (e.g., Litvinenko 1999) and the larger value of $l$ to those in solar active regions (e.g., Litvinenko \& Martin 1999). Observed cancellation events are characterized by convergence speeds of a few hundred $\mathrm{m} \mathrm{s}^{-1}$ (e.g., Litvinenko et al. 2007), which are typical of supergranular flows, implying that the diffusive regime of photospheric cancellation should be easy to detect, especially in smaller canceling features. The problem with an estimate of this sort, however, is that it neglects the likely dependence of both $\tau$ and $\left\langle v^{2}\right\rangle$ on the spatial scale under discussion.

Recently, Chae et al. (2008) analyzed SOHO MDI and Hinode SOT magnetograms, and determined the coefficient of magnetic diffusivity $D$ in the solar photosphere as a function of the magnetogram resolution. Chae et al. (2008) proposed a turbulent scale-dependent diffusivity $D=D_{0} l^{\alpha}$ to explain the highresolution data, specifically

$$
D\left[\mathrm{~cm}^{2} \mathrm{~s}^{-1}\right] \simeq 5 l^{5 / 4}[\mathrm{~cm}]
$$

Chae et al. (2008) pointed out that this empirical scaling relation not only summarizes the high-resolution $\mathrm{SOHO}$ and Hinode data but also reproduces the previously reported values of the diffusivity coefficient determined by granular and supergranular flows (e.g., Smithson 1973; Schrijver \& Martin 1990; Berger et al. 1998; Hagenaar et al. 1999), although some very large reported values of diffusivity are not described by the scaling (e.g., Simon et al. 1995; Giacalone \& Jokipii 2004). In fact, Equation (5) is consistent with the observed diffusivities associated with spatial scales varying by almost two orders of magnitude, with the corresponding values of $D$ ranging between $\simeq 1 \mathrm{~km}^{2} \mathrm{~s}^{-1}$ and $\simeq 200 \mathrm{~km}^{2} \mathrm{~s}^{-1}$. It may be of particular physical interest that the power-law index $\alpha=5 / 4$ in the scaling for $D$ is consistent with the concept that the diffusivity is produced by the Iroshnikov-Kraichnan magnetohydrodynamic turbulence (Iroshnikov 1963; Kraichnan 1965). Indeed, if the correlation time $\tau$ is identified with a turbulent eddy turnover time,

$$
\tau \simeq l /\left\langle v^{2}\right\rangle^{1 / 2}
$$

the turbulent magnetic diffusivity is defined as

$$
D \simeq \tau\left\langle v^{2}\right\rangle
$$

and it follows that

$$
\left\langle v^{2}\right\rangle^{1 / 2} \sim l^{1 / 4}
$$

as in the Iroshnikov-Kraichnan turbulence.

Now Equations (4), (5), and (7) can be combined to give the sought-after criterion for diffusive cancellation:

$$
u l / D<1
$$

or

$$
u\left[\mathrm{~cm} \mathrm{~s}^{-1}\right]<5 l^{1 / 4}[\mathrm{~cm}],
$$

which gives $u<5 \mathrm{~m} \mathrm{~s}^{-1}$ for $l=10^{8} \mathrm{~cm}$ and $u<9 \mathrm{~m} \mathrm{~s}^{-1}$ for $l=10^{9} \mathrm{~cm}$. Thus, diffusive effects can be significant only in canceling features that are characterized by very slow convergence of magnetic fragments. Because the observed cancellation speed $u$ is typically a few hundred $\mathrm{m} \mathrm{s}^{-1}$, the criterion justifies the use of regular cancellation models in interpreting photospheric canceling features (e.g., Litvinenko et al. 2007). The diffusive cancellation time would be as large as $t_{\mathrm{d}} \simeq 10^{5} \mathrm{~s}$ for $l=10^{8} \mathrm{~cm}$, for example.

The dependence of the predicted critical value of $u$ on the fragment size $l$ is a consequence of the turbulent scaling (Equation (8)), which leads to the scaling

$$
t_{\mathrm{d}} \sim l^{2} / D(l) \sim l^{3 / 4}
$$

for the turbulent diffusion time. Because $t_{\mathrm{r}} \sim l$, the condition $t_{\mathrm{d}}<t_{\mathrm{r}}$ cannot be satisfied for smaller canceling magnetic fragments, if the condition is not satisfied for larger fragments. Note for clarity that if the canceling fragments have very small sizes or strongly irregular shapes, resistive diffusion should become more significant than turbulent diffusion. The corresponding length scales, however, are estimated to be as small as $30 \mathrm{~km}$ (Chae et al. 2008).

\section{MAGNETIC FOOTPOINT DIFFUSION IN THE PHOTOSPHERE}

The argument of the previous section relies on the empirical formula (Equation (5)) for the diffusion coefficient $D(l)$. It is natural to ask whether this form of $D$ is meaningful when applied to large-scale dispersal of magnetic flux in the photosphere, as opposed to small-scale flux cancellation considered so far.

Because the diffusion timescale $t_{\mathrm{d}} \sim l^{3 / 4}$ and the regular timescale $t_{\mathrm{r}} \sim l$, it is evident that the magnetic field evolution on sufficiently large spatial scales should be diffusive. Leighton (1964) was the first to interpret the observed dispersal and migration of photospheric magnetic fields as a randomwalk diffusion-like process, caused by supergranular convection. Leighton (1964) assumed a constant value of the diffusion coefficient $D$. For a scale-dependent diffusion coefficient $D(l)$, however, the motion of a footpoint is controlled by a dominant turbulent velocity component on the largest scale. Hence, it is convenient to study the process of relative diffusion, corresponding to the increase in distance between footpoints. Similarly, effects of a regular velocity are subtracted from the data when the diffusion coefficient is derived using a sequence of highresolution magnetograms (Chae et al. 2008). The standard theoretical description of relative diffusion in a turbulent medium is a partial differential equation, motivated by the usual diffusion equation (Richardson 1926), and it is the approach adopted in this Letter (see Chukbar 1993 for alternative approaches).

Consider, therefore, the probability $P(l, t) d l$ for neighboring diffusing magnetic fragments to be separated by a distance 
$l$ after time $t$. For clarity, consider first a one-dimensional diffusion model. On adopting the turbulent diffusion coefficient (Equation (5)) due to Chae et al. (2008),

$$
D(l)=D_{0} l^{5 / 4}
$$

the dispersal of an initially localized photospheric magnetic flux is described by

$$
\frac{\partial P}{\partial t}=\frac{\partial}{\partial l}\left(D_{0} l^{5 / 4} \frac{\partial P}{\partial l}\right)
$$

The normalization of the probability density $P(l, t)$ is given by

$$
\int_{0}^{\infty} P(l, t) d l=1 .
$$

It is worth emphasizing that the turbulent diffusivity $D(l)$ depends on the footpoint separation $l$ rather than position $x$. The probability density $P(l, t)$ is independent of $x$, and the effect of a position-dependent diffusivity is altogether different from the one studied here.

It is clear on dimensional grounds that the typical footpoint separation satisfies

$$
\langle l\rangle \simeq\left(D_{0} t\right)^{4 / 3}
$$

More formally, this scaling follows from the solution to an initial value problem $P(l, 0)=P_{0}(l)$ :

$$
P(l, t)=\int_{0}^{\infty} G(l, \xi, t) P_{0}(\xi) d \xi
$$

where

$$
\begin{aligned}
G(l, \xi, t)= & \frac{4}{3}\left(D_{0} t\right)^{-1}(l \xi)^{-1 / 8} \exp \left[-\frac{16}{9} \frac{\left(l^{3 / 4}+\xi^{3 / 4}\right)}{D_{0} t}\right] \\
& \times I_{1 / 3}\left[\frac{32}{9} \frac{(l \xi)^{3 / 8}}{D_{0} t}\right]
\end{aligned}
$$

is the Green's function, and $I_{1 / 3}$ is a modified Bessel function. For the initial condition $P_{0}(l)=\delta(l)$ or asymptotically for large $t$ for any localized initial distribution $P_{0}(l)$, the probability density simplifies to a self-similar solution

$$
P(l, t)=\left[\left(\frac{3}{4}\right)^{5 / 3} \Gamma\left(\frac{4}{3}\right)\right]^{-1}\left(D_{0} t\right)^{-4 / 3} \exp \left(-\frac{16}{9} \frac{l^{3 / 4}}{D_{0} t}\right),
$$

where $\Gamma$ is the Gamma function, which leads to Equation (15). Generalization of the formal solution for an arbitrary power-law dependence $D(l)=D_{0} l^{\alpha}$ is straightforward.

Similarly, the probability density $P(l, t)$ in two dimensions is found by solving

$$
\frac{\partial P}{\partial t}=\frac{\partial}{\partial l}\left[D_{0} l^{9 / 4} \frac{\partial}{\partial l}\left(\frac{P}{l}\right)\right],
$$

and a self-similar solution in this more realistic case is given by

$$
P(l, t)=\left[\left(\frac{3}{4}\right)^{13 / 3} \Gamma\left(\frac{8}{3}\right)\right]^{-1}\left(D_{0} t\right)^{-8 / 3} l \exp \left(-\frac{16}{9} \frac{l^{3 / 4}}{D_{0} t}\right),
$$

which again leads to Equation (15).
Following Leighton (1964), observers typically assume the footpoint diffusion coefficient to be a constant (e.g., Schrijver \& Martin 1990; Hagenaar et al. 1999). It is well known that essentially the same diffusion equation describes the usual displacement probability density and $P(l, t)$ if $D=$ const (Richardson 1926), in which case the diffusion coefficient is equal to $\left\langle l^{2}\right\rangle / t$ times a numerical coefficient. By contrast, the quantity $\left\langle l^{2}\right\rangle / t$ cannot be a constant if $D=D(l)$. In fact, it follows from Equation (15) that

$$
\left\langle l^{2}\right\rangle / t \sim t^{5 / 3}
$$

This scaling agrees, at least qualitatively, with the results of Hagenaar et al. (1999) who analyzed SOHO MDI magnetograms and concluded that the diffusion coefficient appeared to be an increasing function of the time interval for which it was measured. Hagenaar et al. (1999) ascribed this result to diffusion driven by random flows on both the granular and supergranular scales. While little is known about the dynamics on the intermediate scales, Hagenaar et al. (1999) also hypothesized the presence of an additional, much larger diffusive scale.

The concept that random displacements of magnetic footpoints occur on several scales, advocated by Hagenaar et al. (1999), is consistent with the analysis of Chae et al. (2008), who argued that the observed diffusion reflects the presence of a turbulent spectrum of fluctuations of the photospheric velocity and magnetic fields. What remains unclear though is whether the random flows that drive diffusion occur on distinct or overlapping scales. In the latter case, a continuous approximation for the diffusion coefficient $D(l)$, adopted in this Letter, should be more accurate.

\section{DISCUSSION}

The diffusive regime of photospheric cancellation has not yet been analyzed as thoroughly as regular cancellation either observationally or theoretically. Such analyses will be required in order to gain a better understanding of the relative roles of diffusive and regular processes in photospheric cancellation. Although small-scale fluctuating components of the photospheric velocity are averaged out in the data, they lead to an effective diffusion of magnetic flux concentrations.

As argued in this Letter, two related estimates suggest that diffusive effects play a relatively minor role in photospheric cancellation. First, the observed cancellation speeds are typically too fast to be explained by magnetic field diffusion in the photosphere. Second, the dependence of the diffusive cancellation time on the length scale $t_{\mathrm{d}} \sim l^{3 / 4}$ means that diffusive cancellation cannot occur on smaller scales either. As usual, magnetic reconnection corresponds to a balance between resistive and advective effects, and so resistive diffusion becomes more significant than turbulent diffusion on very small length scales.

Therefore, a somewhat surprising conclusion is that regular cancellation models (e.g., Litvinenko et al. 2007; Park et al. 2009) should be expected to give a quantitatively accurate description of photospheric cancellation.

While the observed diffusivity $D(l)$ is consistent with the Iroshnikov-Kraichnan turbulent spectrum (Chae et al. 2008), the spectral slope generally is expected to depend on the strength of the large-scale magnetic field that corresponds to the axial field in solar coronal loops (Matthaeus \& Zhou 1989). As noted by the referee, theoretical models of anisotropic magnetohydrodynamic spectral transfer have been developed 
(e.g., Zhou \& Matthaeus 1990; Matthaeus et al. 2009; Galtier \& Buchlin 2010), which are based on diffusion approximation (Leith 1967). It is potentially important that more detailed measurements of the photospheric magnetic diffusivity may provide new observational constraints for the theoretical models.

Finally, it should be noted that the arguments of this Letter rest on the description of turbulent diffusion that goes back to Richardson (1926). Hence, the problem may have to be reconsidered if, for instance, anomalous diffusion models turn out to give the correct description of the photospheric magnetic field evolution (Ruzmaikin et al. 1996; Cadavid et al. 1999; cf. Giacalone \& Jokipii 2004).

This work was initiated during a visit to Seoul National University. Numerous useful discussions with Professor Jongchul Chae are gratefully acknowledged. The work was supported by NSF grants ATM-0734032 and ATM-0837915 and by a research fellowship from the Alexander von Humboldt Foundation.

\section{REFERENCES}

Berger, T. E., Löfdahl, M. G., Shine, R. A., \& Title, A. M. 1998, ApJ, 506, 439 Cadavid, A. C., Lawrence, J. K., \& Ruzmaikin, A. A. 1999, ApJ, 521, 844

Chae, J., Litvinenko, Y. E., \& Sakurai, T. 2008, ApJ, 683, 1153

Chae, J., Moon, Y.-J., Wang, H., \& Yun, H. S. 2002, Sol. Phys., 207, 73

Chukbar, K. V. 1993, J. Exp. Theor. Phys. Lett., 58, 90

Galtier, S., \& Buchlin, É. 2010, ApJ, 722, 1977

Giacalone, J., \& Jokipii, J. R. 2004, ApJ, 616, 573
Hagenaar, H. J., Schrijver, C. J., Title, A. M., \& Shine, R. A. 1999, ApJ, 511, 932

Iroshnikov, R. S. 1963, Astron. Zh., 40, 742

Kraichnan, R. H. 1965, Phys. Fluids, 8, 1385

Leighton, R. B. 1964, ApJ, 140, 1547

Leith, C. E. 1967, Phys. Fluids, 10, 1409

Litvinenko, Y. E. 1999, ApJ, 515, 435

Litvinenko, Y. E. 2010, ApJ, 720, 948

Litvinenko, Y. E., Chae, J., \& Park, S.-Y. 2007, ApJ, 662, 1302

Litvinenko, Y. E., \& Martin, S. F. 1999, Sol. Phys., 190, 45

Livi, S. H. B., Wang, J., \& Martin, S. F. 1985, Aust. J. Phys., 38, 855

Martens, P. C., \& Zwaan, C. 2001, ApJ, 558, 872

Martin, S. F. 1990, in IAU Symp. 138, Solar Photosphere: Structure, Convection, and Magnetic Fields, ed. J. O. Stenflo (Dordrecht: Kluwer), 129

Martin, S. F. 1998, Sol. Phys., 182, 107

Martin, S. F., Livi, S. H. B., \& Wang, J. 1985, Aust. J. Phys., 38, 129

Martin, S. F., Panasenco, O., Engvold, O., \& Lin, Y. 2008, Ann. Geophys., 26, 3061

Matthaeus, W. H., Oughton, S., \& Zhou, Y. 2009, Phys. Rev. E, 79, 035041

Matthaeus, W. H., \& Zhou, Y. 1989, Phys. Fluids B, 1, 1929

Okamoto, T. J., Tsuneta, S., \& Berger, T. E. 2010, ApJ, 719, 583

Park, S., Chae, J., \& Litvinenko, Y. E. 2009, ApJ, 704, L71

Parker, E. N. 1957, J. Geophys. Res., 62, 509

Richardson, L. F. 1926, Proc. R. Soc. A, 110, 709

Ruzmaikin, A. A., Cadavid, A. C., Chapman, G., Lawrence, J. K., \& Walton, S. 1996, ApJ, 471, 1022

Schrijver, C. J., \& Martin, S. F. 1990, Sol. Phys., 129, 95

Simon, G. W., Title, A. M., \& Weiss, N. O. 1995, ApJ, 442, 886

Smithson, R. C. 1973, Sol. Phys., 29, 365

Sturrock, P. A. 1999, ApJ, 521, 451

Sturrock, P. A., \& Uchida, Y. 1981, ApJ, 246, 331

Wang, Y.-M., \& Muglach, K. 2007, ApJ, 666, 1284

Wood, P., \& Martens, P. C. 2003, Sol. Phys., 218, 123

Zhou, Y., \& Matthaeus, W. H. 1990, J. Geophys. Res., 95, 14881 